\title{
Will ET Write or Radiate: inscribed matter vs. electromagnetic channels
}

\author{
Christopher Rose $^{1 *}$ and Gregory Wright ${ }^{2}$ \\ ${ }^{1}$ Department of Electrical and Computer Engineering, Rutgers University, \\ Piscataway, NJ 08854, USA \\ ${ }^{2}$ Antiope Associates, \\ 18 Clay Street \\ Fair Haven, NJ 07704 \\ *To whom correspondence should be addressed; E-mail: crose@winlab.rutgers.edu.
}

\begin{abstract}
We consider the energy requirements of information carriage using physical transport of inscribed matter and compare it to that using electromagnetic radiation when delivery delay beyond light transit time can be tolerated. Somewhat counter-intuitively, physical transport of inscribed matter is often more energy efficient than electromagnetic broadcast by many orders of magnitude over a wide range of scenarios - from chipto-chip computer communications to interstellar signaling. In fact, the efficiencies are so enormous that it may even be more likely for initial contact by extraterrestrial civilizations to occur using physical artifacts - essentially messages in a bottle - than via electromagnetic communication.
\end{abstract}

\section{INTRODUCTION}

At one time or another, every communication theorist has had the following epiphany:

Driving a truck filled with storage media (books, DVDs, tapes, etc.) across town constitutes a very reliable channel with an extremely large bit rate.

Tannenbaum mentions the possibility in his communications textbook [1] and so does Rolf Landauer regarding inscription and physical transport in the context of reversible communication [2]. Similarly, Gray et al [3] consider the viability of information carriage through transport of mass storage media from an economic perspective.

Our epiphanies have occurred a few times over many years, but most recently with the study of short range high data rate channels [4]-[13] and mobility assisted wireless networks [14], [15] where communications nodes only transfer data to one another when the channel is good - typically at close range. One natural extension of this work is to not radiate electromagnetic energy at all, but rather, to have nearby nodes physically exchange "letters" inscribed on some medium. And from such imaginings comes a simple question: when is it better to write than to radiate?

We will show that under a surprising variety of circumstances letters are, bit for bit, much more energy efficient than methods based on electromagnetic radiation when delay beyond light transit time can be tolerated. Moreover, from a theoretical perspective, the cost of writing the information into some medium can be made infinitessimal at arbitrary rate [2], [16], [17], so the energy savings are not necessarily diminished by adding the inscription or readout costs. Thus, something seemingly so primitive as hurling carved pebbles through space can require many orders of magnitude less energy than broadcasting the same information.

\section{ENERGY CALCULATIONS}

Our argument requires calculation of energy budgets for "inscribed matter" and electromagnetic communication under the scenario depicted in FIGURE 1.

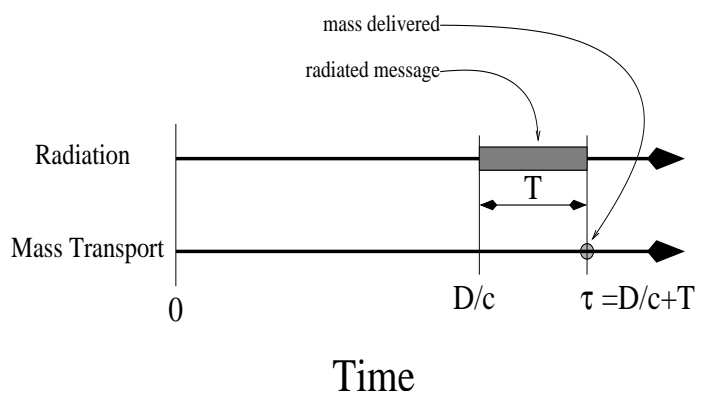

Fig. 1. A message of $B$ bits is sent over a distance $D$ and received by a deadline, $\tau$. In the case of radiation, the transmission is of duration $T$, so the entire message is available at the receiver after a delay of $\tau=D / c+T$. In this way, the standard measure of communication effi ciency, bits per joule, applies to both the electromagnetic radiation and inscribed matter cases thereby allowing direct comparison. We assume the destination is at rest relative to the source and that the receiver will capture the inscribed matter message with an additional energy expenditure that need not be greater than the launch energy.

The energy required to deliver inscribed matter a distance $D$ by deadline $\tau$ in free space is minimized if the particle is launched at speed $\bar{v}=D / \tau$. This result is well known but can also be derived simply using standard communication theory tools such as Jensen's inequality [18]. We find it convenient to parameterize the acceptable delay by a dimensionless quantity $\delta=c \tau / D$ where $\delta \approx 1$ means that we require the message to be available at a time just greater than the light transit delay, and $\delta \gg 1$ means we can tolerate a long delay. Thus, for a message of size $B$ using matter with "mass information density" $\tilde{\rho}$ (bits 
$\mathrm{kg}^{-1}$ ) we have

$$
E_{w}=\frac{1}{2} m_{B} \bar{v}^{2}=\frac{1}{2} \frac{B}{\tilde{\rho}}\left(\frac{c}{\delta}\right)^{2} .
$$

Notice that we assume messages travel at non-relativistic speeds. That is, relativistic effects are only important when $v \gtrsim 0.7 c$, and by our original assumption, we are willing to accept delays much greater than light transit time.

We also note that particle delivery might require overcoming a potential field (gravity), but carefully computing its effect will not greatly affect our main points. For example, terrestrial travel requires an initial upward velocity (and associated energy expenditure). This energy is minimized when the forward velocity and initial upward velocity are equal - the usual artillery problem. We note that for a given gravitational field and target range, this minimum energy solution implies a corresponding "natural" forward speed and associated delay $\delta^{*}$ which will be calculated later.

For interstellar travel, we must add an escape energy proportional to $v_{e}^{2}$ where $v_{e}$ is the escape velocity. Thus, if we require $\bar{v}=\alpha v_{e}$ where $\bar{v}$ is the required velocity far from the gravitational well and $\alpha$ is some constant, then the energy penalty factor is $1+1 / \alpha^{2}=1.25$ when $\alpha=2$.

The energy used to send an electromagnetic message is $P T$ where $P$ is the radiated power. A receiver at some distance $D$ will capture some fraction of this power $\mathrm{v}(D) P$ where $\mathrm{v}(D)$ is defined as the energy capture coefficient of the receiver. We assume square law isotropic propagation loss with transmitting antenna gain $G$

$$
G_{\max }=\frac{8 \pi^{2} R^{2}}{\lambda^{2}}=2 \pi^{2} \mathcal{A}^{2}
$$

for diffraction limited beams from a circular filled aperture of radius $R$ operating at a wavelength $\lambda$ [19]. The normalized aperture, $\mathcal{A}=2 R / \lambda$ is the aperture in units of the wavelength. Equation (2) is only valid in the far-field, meaning distances at which the wavefronts are essentially spherical. We then have $\mathrm{v}(D)=G A / 4 \pi D^{2}$ where $A$ is the effective collecting area of the receiver.

Then, assuming additive Gaussian receiver noise, the Shannon capacity [20] in bits per second between the transmitter and receiver is

$$
C=W \log _{2}\left(\frac{P G A}{4 \pi D^{2} N_{0} W}+1\right)
$$

where $N_{0}$ is the background noise spectral intensity and $W$ is the channel bandwidth. If we assume a transmission interval long enough that the usual information theoretic results for long codes can be applied, the number of bits delivered for a transmission of duration $T$ is $B=T C$. Notice that we have set the time required for the arrival of the complete message to $\tau=D / c+T$, identical to the inscribed matter deadline shown in FIGURE 1.

Since $E_{r}=P T$, we bound $E_{r}$ from below by assuming a large "time-bandwidth" product $T W$ to obtain

$$
E_{r} \geq B N_{0} \frac{4 \pi D^{2}}{A G} \ln 2
$$

In so doing, we consider a best case scenario with unbounded degrees of communication freedom. Thus, our energy estimate is conservative since any method of electromagnetic communication cannot use less energy than given in equation (4).

We define $\Omega$, the radiation to transport energy ratio, as

$$
\Omega=\frac{E_{r}}{E_{w}}
$$

which by combining equation (1) and equation (4) yields

$$
\Omega \geq\left[\frac{\tilde{\rho} N_{0}}{c^{2}}\right]\left[(2 \ln 2) \delta^{2}\right]\left[\frac{4 \pi D^{2}}{A G}\right] .
$$

Considering the case where the transmitting and receiving antennas are the same size, equation (6) becomes

$$
\Omega \geq \frac{16 \ln 2}{\pi^{2}}\left[\frac{\tilde{\rho} N_{0}}{c^{2}}\right]\left[\frac{\mathcal{D}}{\mathcal{A}}\right]^{2} \delta^{2},
$$

where $\mathcal{A}=2 R / \lambda$ is the normalized antenna aperture and $\mathcal{D}=$ $D / 2 R$ is the distance between the transmitter and receiver in units of the antenna aperture.

From equation (6) we can also calculate the minimum value of mass information density $\tilde{\rho}^{*}$ such that inscribed matter will be more efficient than radiation even when radiation is perfectly focused at the target

$$
\tilde{\rho}^{*}=\frac{1}{2 \ln 2}\left[\frac{c^{2}}{N_{0}}\right] \delta^{-2} .
$$

\section{EMPIRICAL MASS INFORMATION DENSITIES}

Calculating the energy required to deliver an inscribed matter message requires knowing how densely information can be packed - the aforementioned mass information density $\tilde{\rho}$ in bits $\mathrm{kg}^{-1}$. Absolute bounds on mass information density have been described in [22], but these limits assume matter in its densest possible state (a black hole) and are far larger than what can be obtained practically with ordinary matter. In contrast, clear limits on the maximum possible information storage density for ordinary inscribed matter are unknown.

It is therefore useful to consider current empirical limits on mass information density. A scanning tunneling microscope (STM) can place an equivalent of about $10^{15}$ bits per square inch using individual xenon atoms on a nickel substrate [23]. The per bit dimension is then $0.8 \mathrm{~nm}$ on a side. Assuming a $100 \AA$ nickel buffer between layers we obtain a bit density of $1.56 \times 10^{20}$ bits $\mathrm{cm}^{-3}$, or

$$
\tilde{\rho}_{\mathrm{STM}}=1.8 \times 10^{22} \text { bits } \mathrm{kg}^{-1} \text {. }
$$

If we could build stable alloys of the lightest solid elements (Li, Be) with arbitrary placement of the atoms, we could achieve

$$
\tilde{\rho}_{\mathrm{LiBe}}=7.5 \times 10^{25} \text { bits } \mathrm{kg}^{-1} \text {. }
$$

For comparison, the information density of single-stranded RNA (e.g., polio virus RNA) is about $3.6 \times 10^{24}$ bits $\mathrm{kg}^{-1}$.

E-beam lithography on silicon can achieve feature sizes of $5 \mathrm{~nm}$ which implies a bit density of $4 \times 10^{12}$ bits $\mathrm{cm}^{-2}$. Assuming $100 \AA$ substrate layers we then have $4 \times 10^{18}$ bits 
$\mathrm{cm}^{-3}$. Given silicon's density of $2.6 \mathrm{~g} \mathrm{~cm}^{-3}$ [24] the mass information density is

$$
\tilde{\rho}_{\mathrm{e}-\text { beam }}=1.54 \times 10^{21} \mathrm{bits} \mathrm{kg}^{-1},
$$

about three orders of magnitude smaller than RNA.

Current optical lithographic techniques routinely achieve 0.1 $\mu \mathrm{m}$ feature sizes. Assuming a substrate thickness of $100 \AA$ this corresponds to a density of 10000 bits $\mu \mathrm{m}^{-3}$ or $10^{13}$ bits $\mathrm{mm}^{-3}$. Again assuming that silicon is the substrate material gives

$$
\tilde{\rho}_{\text {optical }}=3.85 \times 10^{18} \text { bits kg }^{-1},
$$

or approximately six orders of magnitude smaller than RNA.

Magnetic storage density is on the order of $10 \mathrm{~Gb} \mathrm{~cm}^{-2}$ so that again, each bit is about $0.1 \mu \mathrm{m}$ on a side. Assuming a film thickness of $1000 \AA$ and a density similar to $\mathrm{FeO}_{2}$ (about $5 \mathrm{~g} \mathrm{~cm}^{-3}$ [24]) we have

$$
\tilde{\rho}_{\text {magnetic }}=2 \times 10^{17} \text { bits kg }^{-1},
$$

which is about seven orders of magnitude smaller than RNA.

More pedestrian forms of information storage include DVDs at about

$$
\tilde{\rho}_{\text {DVD }}=3 \times 10^{12} \text { bits kg }^{-1}
$$

(or $3 \times 10^{15}$ bits kg $^{-1}$ if $1 \mu \mathrm{m}$ laminated layers could be used), and even laser-printed paper at 1000 dots per inch has a mass information density of approximately

$$
\tilde{\rho}_{\text {paper }}=10^{10} \text { bits kg }^{-1} \text {. }
$$

Finally, consider that the Voyager spacecraft carry plaques bearing inscribed messages - pictograms, images and audio recordings - encoded as on a phonograph record and including a stylus with which to play it. If we assume that the total information content of these messages is under $10^{9} \mathrm{bits}$, then at a total weight of approximately one ton (about 909 $\mathrm{kg}$ ) [25], the mass information density of the Voyager craft is approximately

$$
\tilde{\rho}_{\text {voyager }}=1.1 \times 10^{6} \mathrm{bits} \mathrm{kg}^{-1} \text {. }
$$

\section{COMmunication Scenarios}

\section{Chip-to-Chip Messaging}

There are a variety of scenarios where information must be shuttled between processors. Networks of sensor "motes" come to mind as do multiprocessor parallel computers. To proceed we first calculate the minimum energy delay, $\delta^{*}$, by noting that a particle with initial upward velocity $v$ will return to its vertical starting point at time $\tau=2 v / g$. To reach the target by time $\tau$ the initial forward velocity must therefore be $D g / 2 v$ which results in $v=D g / 2 v$ owing to initially equal forward and upward velocities. The result is

$$
\delta^{*}=\frac{c}{\sqrt{D g / 2}}
$$

which allows equation (7) to be rewritten as

$$
\Omega \geq \frac{16 \ln 2}{\pi^{2}}\left[\frac{\mathcal{D}}{\mathcal{A}^{2}}\right] \frac{\tilde{\rho} N_{0}}{R g} .
$$

Owing to size and antenna gain considerations, communication at optical frequencies is preferred. So, consider a system with processors separated by $D=10 \mathrm{~cm}$ and infrared $(\lambda=1 \mu \mathrm{m})$ laser apertures of radius $R=5 \mu \mathrm{m}$. The normalized distance is then $\mathcal{D}=10^{4}$ and the normalized aperture is $\mathcal{A}=10$. If we assume a receiver temperature of $300 K$ and that magnetic film "chits" with $\tilde{\rho} \approx 10^{17}$ bits $\mathrm{kg}^{-1}$ could be used as the transport medium, we have $\Omega \geq 930$. (For $D=1 \mathrm{~m}$ we would have $\Omega \geq 9300$.)

Thus, inscribed matter is about 1000 times more energy efficient than free space IR laser communication. However, we note that with significantly larger (receive and transmit) apertures virtually all the radiated energy could be focused on the target so that by equation (8) an inscription medium with $\tilde{\rho} \approx 5 \times 10^{20}$ would be needed to make inscribed matter more efficient than radiation in this example.

\section{Terrestrial Messaging}

Now consider a typical terrestrial wireless scenario with hand-held mobile units. Assume an aperture of $R=5 \mathrm{~cm}$, an operating frequency of $3 \mathrm{GHz}(\lambda=10 \mathrm{~cm})$ and as before, a receiver temperature of $300 \mathrm{~K}$. With $\tilde{\rho}=10^{17}$, from equation (18) we have $\Omega \geq 0.046$ for a range of $10 \mathrm{~m}$ and $\Omega \geq 4.6$ for a range of 1000 meters. We note that the radiation calculation is based on free space propagation. Were the more typical $D^{4}$ terrestrial propagation loss assumed, the $\Omega$ bounds would increase to 4.6 and $4.6 \times 10^{6}$ respectively.

It is interesting to note that ballistic transport at minimum energy between New York City and Boston requires about 250 seconds - not terribly much delay. What if greater delays were tolerable? As one might expect from the derivation, greater delays impose greater and greater energy requirements on ballistic transport since larger and larger vertical velocities are necessary to delay return to the launch altitude, so let us consider automobile delivery.

A typical automobile burns 1 gallon of fuel per 20 miles traveled. Since gasoline liberates about $1.2 \times 10^{8}$ Joules [26, pp.317] per gallon, the NY-Boston trip would consume $2.4 \times$ $10^{10}$ Joules. Assume a $100 \mathrm{~kg}$ payload of DVDs with a total information content of $3 \times 10^{14}$ bits - about the size of text information in the Library of Congress. Assuming $D^{4}$ isotropic propagation, a receiver temperature of $300 \mathrm{~K}$ and a receiver aperture of $10 \mathrm{~m}^{2}$, the corresponding radiated energy necessary to deliver the message is about $10^{16}$ Joules. So even in this simple scenario where relatively inefficient physical transport is used, inscribed matter beats radiation by a factor of about $4 \times 10^{5}$. Even more interesting, if laser-printed paper were used (equation (15)), the factor would still be 400 .

We then note that even using gigabit fiber links, this transfer would take 300,000 seconds (about 3.5 days). So it not too surprising that businesses like Federal Express and Netflix are viable.

\section{Interstellar Messaging}

The interstellar messaging problem was considered in detail elsewhere [27]. Here we summarize the results. 
In FIGURE 2 we provide iso- $\Omega$ contours as a function of $\mathcal{D}$ and $\mathcal{A}$ for communications with $\delta=10^{3}$ ( $\bar{v}$ about 7 times larger than solar escape velocity at earth distance) and $\tilde{\rho}=10^{22}$. Points corresponding to examples of receiver apertures and wavelengths at various distances are as shown. We see that inscribed matter is energetically favored over a wide range of conditions. For instance, inscribed matter is more efficient than radiation between Arecibo-sized apertures for distances greater than $1.32 \times 10^{12} \mathrm{~m}\left(\mathcal{D}=4.4 \times 10^{9}\right)$, roughly the distance from the Sun to Saturn.

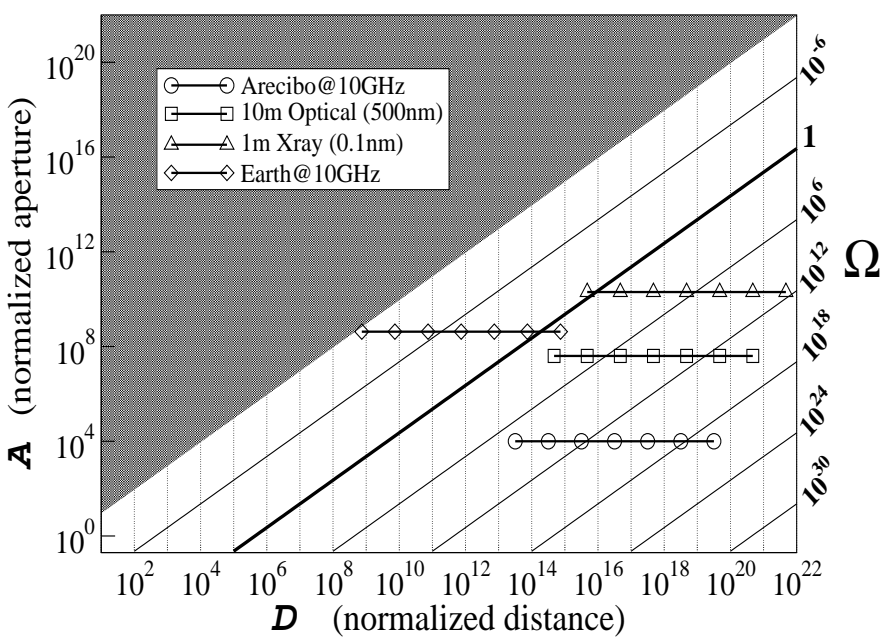

Fig. 2. Effi ciency $(\Omega)$ of inscribed matter compared to electromagnetic communication. Contours of constant $\Omega$ are plotted as a function of $\mathcal{A}=2 R / \lambda$ (a measure of transmit antenna directivity) and $\mathcal{D}=D / 2 R$ (a measure of receiver power capture) using equation (7). We assume equal-sized transmit and receive apertures, receiver noise temperature $3 \mathrm{~K}, \delta=10^{3}$ and $\tilde{\rho}=10^{22}$ bits $\mathrm{kg}^{-1}$. Below and to the right of the line labeled $\Omega=1$, inscribed mass is a more energetically effi cient means of communication. In the shaded region, aperture and distance are such that no additional advantage is conferred by tighter beam collimation - all radiated energy is captured at the target. In this parameterization, isotropic transmission is given by $\mathcal{A}=1 / \pi \sqrt{2}$, the smallest value of ordinate plotted. $(\mathcal{D}, \mathcal{A})$ points are also shown for various antenna aperture sizes (Arecibo, $R=150 \mathrm{~m}$, an antenna the diameter of the Earth, $R=6.38 \times 10^{6} \mathrm{~m}, 10 \mathrm{~m}$ optical and $1 \mathrm{~m} \mathrm{X}$-ray telescopes) and radiation wavelengths $0.03 \mathrm{~m}(10 \mathrm{GHz}), 500 \mathrm{~nm}$ (optical) and $0.1 \mathrm{~nm}$ (X-ray). Target ranges from 1 to $10^{6}$ light years in $\times 10$ light year increments are depicted for each case. For reference we note that $\delta=7143$ at solar escape velocity near earth $\left(42 \mathrm{~km} \mathrm{sec}^{-1}\right)$ while here we use a speedier $\delta=10^{3}$.

It is interesting to note that the advantage extends down to very low mass information densities as well. Consider the Voyager spacecraft which had it been fired from a catapult would have an information energy efficiency of about 800 Joule bit ${ }^{-1}$. Thus, Voyager is more efficient than AreciboArecibo radio communications beyond about 2000 light years, and if instead of plaques, the Voyager craft had carried a few DVDs, this breakeven distance would be 200 light years.

Only between truly enormous earth-sized microwave apertures does radiation remain more efficient at large distances; e.g., across the Milky Way. But at sufficiently large distance, inscribed matter is always eventually more efficient than radiation owing to the unavoidable $D^{-2}$ dispersal of radiation from a fixed size aperture.

Of course, $D^{-2}$ dispersal of electromagnetic radiation can be a virtue for broadcast messages since using the same energy, many targets will be illuminated. In contrast, if $N$ targets are chosen, inscribed matter requires $N$ separate mass packets and therefore an $N$-fold increase in energy. To make the comparison between radiation and inscribed matter we assume the stellar density of the Milky Way $\left(\eta=2.8 \times 10^{-2}\right.$ stars (light year) ${ }^{-3}$ ) and overestimate the inscribed matter energy budget by assuming the same velocity is used to reach nearby targets as more distant ones.

For a distance $D$, a wavelength $\lambda$ and transmitter aperture $R_{t}$, the far-field beam radius is approximately $2 D / \mathcal{A}$. so that the volume inside the beam is roughly $4 \pi D^{3} / 3 \mathcal{A}^{2}$. Thus, the average number of stars illuminated is

$$
N=3.73 \times 10^{-2} \pi\left[\frac{D}{\text { light year }}\right]^{3} \frac{1}{\mathcal{A}^{2}}
$$

with $D$ measured in light years. For the Arecibo example at ten thousand light years we have a penalty of $N \approx 1000$ which is inconsequential given the inscribed matter advantage of $5 \times$ $10^{15}$ seen in FIGURE 2.

Next consider isotropic radiation where many more targets are covered and assume a spherical galaxy with Milky Way stellar density. At a range of $10^{4}$ light years, the number of targets is $N=1.2 \times 10^{11}$ - which seems substantial, but is dwarfed by the inscribed matter advantage of approximately $10^{25}$. (See FIGURE 2 for an isotropic antenna, $\mathcal{A}=1 / \pi \sqrt{2}$.) At a range of $10^{6}$ light years, $N=1.2 \times 10^{17}$ while the inscribed matter advantage is $10^{29}$. Thus, at Milky Way stellar densities, even were every star targeted instead of only a likely subset [28], [29], inscribed matter would still be much more efficient than radiation.

\section{CONCLUSION}

Inscribed matter channels can be many many orders of magnitude more energy efficient than channels which use electromagnetic radiation for distances both large and small. For smaller distances, perhaps distributed multiprocessor machine design could also reap some benefits from inscribed matter communication methods. For moderate distance "existence proofs" we might consider the success of services offered by companies such as NetFlix and FedEx.

In previous work [27], various factors which would affect the efficiency of inscribed matter transmission over interstellar distances were considered. The most important of these included deceleration at the target and shielding of a message from cosmic rays for a long voyage. For a $10^{4}$ light year trip, the overall penalty was about $10^{7}$ - insufficient to overcome the inscribed matter advantage.

Of course, we have left somewhat open the issue of message detection at target. That is, inscribed matter messages must be found among a sea of matter at the target solar system. The formal detection problem is difficult to formulate for a variety of reasons including the possibility that messages could actively use local energy resources for both placement 
and advertisement of their existence. Also consider that even if we assume a listener will always detect a given radiated message, the message must be repeated since the target might not be listening when the message arrives. Depending upon the model used for the emergence and persistence of listeners, large numbers $\left(10^{4}\right)$ of repetitions might be necessary to assure that the message was received [27]. So at a minimum, one could imagine sending multiple copies of a given message to each target without diminishing the inscribed matter advantage derived here.

Finally, we have completely skirted the issue of what sort of messages one might want to send or where they might be sent [28]-[31]. For example, it seems likely that the delays associated with interstellar travel and the seeming fragility of civilization to cosmic (and perhaps self-inflicted) insults suggests that messages should be archival knowledge repositories constructed primarily "for posterity" (cultural or biological preservation) as opposed to solely for initiating a chat. In a similar vein, one could imagine "colonization" as a goal via some process akin to panspermia [32]. Regardless of possible motives, the apparent superiority of inscribed matter over electromagnetic message transmission suggests that we more carefully consider local search for extraterrestrial artifacts. Furthermore, the possibility of purposeful interstellar inscribed matter transmission raises interesting questions about terrestrial biological history.

\section{ACKNOWLEDGMENTS}

We wish to thank G.J. Foschini and Paul Henry for many useful conversations, Peter Shor for pointing out Rolf Landauer's study of reversible computation and communication, Guillermo Gonzalez for citations on bacterial viability in space (and for habitable zone citations) and Stavros Toumpis for his careful reading of an earlier manuscript. Finally we thank Norman Beaulieu for editorial encouragement.

\section{REFERENCES}

[1] Andrew S. Tannenbaum. Computer Networks. Prentice Hall, 2002.

[2] Rolf Landauer. Energy Requirements in Communications. Appl. Phys. Lett, 51(24):2056-2058, 1987.

[3] J. Gray, W. Chong, Barclay T, A. Szalay, and J. vandenBerg. TeraScale SneakerNet: Using Inexpensive Disks for Backup, Archiving, and Data Exchange. Technical Report MSR-TR-2002-54, Microsoft Research, May 2002. (http://research.microsoft.com/research/pubs).

[4] D. J. Goodman, J. Borras, N. B. Mandayam, and R. D. Yates. INFOSTATIONS : A New System Model for Data and Messaging Services In Proceedings of IEEE VTC'97, volume 2, pages 969-973, May 1997. Phoenix, AZ.

[5] J. Borras and R. D. Yates. Infostations overlays in cellular systems. In Proceedings of the Wireless Communications and Networking Conference, WCNC, volume 1, pages 495-499, 1999.

[6] J. Irvine, D. Pesh, D. Robertson, and D. Girma. Effi cient UMTS Data Service Provision using Infostations. In Proceedings of the IEEE Vehicular Technology Conference, volume 3, pages 2119-2123, 1998.

[7] A. Iacono and C. Rose. Bounds on fi le delivery delay in an infostations system. In Vehicular Technology Conference, pages 6.11-3, May 2000. Tokyo.

[8] H. Mao, G. Wu, C.-W. Chu, J. Evans, and M. Caggiano. An Adaptive Radio Link Protocol for Infostations. In Proceedings of the IEEE Vehicular Technology Conference, volume 2, pages 1345-1349, 1999.

[9] H. Mao, G. Wu, C.-W. Chu, J. Evans, and M. Caggiano. Performance evaluation of radio link protocol for infostations. Vehicular Technology Conference, 1999
[10] J. Irvine and D. Pesh. Potential of DECT Terminal Technology for Providing Low-cost Wireless Internet Access through Infostations. In IEE Colloquium on UMTS Terminal and Software Radio, pages 12/1-6, 1999.

[11] A. Iacono and C. Rose. Infostations: New Perspectives On Wireless Data Networks. In Next Generation Wireless Networks. Kluwer Academic, May 2000. Editor: S. Tekinay.

[12] R. H. Frenkiel, B. R. Badrinath, J. Borras, and R. Yates. The Infostations Challenge: Balancing Cost and Ubiquity in Delivering Wireless Data. IEEE Personal Communications, 7(2):66-71, April 2000.

[13] A. Iacono and C. Rose. MINE MINE MINE: Information Theory, Infostation Networks and Resource Sharing. In WCNC 2000, September 2000. Chicago, IL.

[14] M. Grossglauser and D. Tse. Mobility Increases the Capacity of Wireless Ad Hoc Networks. In IEEE Infocom, 2001.

[15] .S. Toumpis and A.J. Goldsmith. Capacity Regions For Wireless Ad Hoc Networks. In Proc. IEEE ICC'O2, April 2002. (also available at http://wsl.stanford.edu/Publications/Stavros/icc02.pdf).

[16] Rolf Landauer. Computation, Measurement, Communication and Energy Dissipation. In Selected Topics in Signal Processing, pages 18-47. Prentice-Hall, 1989. S. Haykin, Ed.

[17] Rolf Landauer. Minimal Energy Requirements in Communication. Science, 272(5270):1914-1918, 1996.

[18] C. Rose. Write or Radiate? In IEEE Fall Vehicular Technology Conference, October 2003. Orlando, FL.

[19] Merrill Skolnik. Introduction to Radar Systems. McGraw-Hill, 2002.

[20] T.M. Cover and J.A. Thomas. Elements of Information Theory. WileyInterscience, 1991.

[21] G. Cocconi and P. Morrison. Searching for Interstellar Communications. Nature, 184:844-846, September 1959.

[22] J.D. Bekenstein and M. Schiffer. Quantum Limitations on the Storage and Transmission of Information. Int. J. Mod. Phys C, 1:355-422, 1990.

[23] D. M. Eigler and E. K. Schweizer. Positioning Single Atoms with a Scanning Tunnelling Microscope. Nature, 344:524-526, 1990.

[24] R.C. Weast. Handbook of Chemistry and Physics. CRC Press, Boca Raton, 1985. 65th Edition.

[25] NASA. http://voyager.jpl.nasa.gov/mission/didyouknow.html.

[26] URL: http://tonto.eia.doe.gov/FTPROOT/multifuel/038497.pdf.

[27] C. Rose and G. Wright. Inscribed Matter As An Energy-Effi cient Means Of Communication With An Extraterrestrial Civilization. Nature, 431:47-49, 2004

[28] G. Gonzalez, D. Brownlee, and P.D. Ward. The Galactic Habitable Zone I: Galactic Chemical Evolution. Icarus, 152(1):185-200, July 2001.

[29] C.H. Lineweaver. An Estimate of the Age Distribution of Terrestrial Planets in the Universe: Quantifying Metalicity as a Selection Effect. Icarus, 151(2):307-313, June 2001.

[30] Bela A. Balas. SETI and the Galactic Belt of Intelligent Life. In Bioastronomy 99: A New Era in Bioastronomy, August 1999.

[31] G. Gonzalez, D. Brownlee, and P.D. Ward. Refuges for Life in a Hostile Universe. Scientific American, page 60, October 2001.

[32] F.H.C. Crick and L.E. Orgel. Directed Panspermia. Icarus, 19:341, 1973. 